\title{
Inhaltsverzeichnis
}

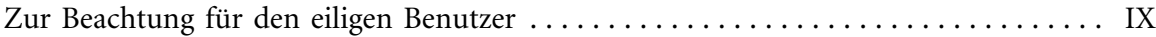

Regeln für die Bildung und den Gebrauch von Abkürzungen $\ldots \ldots \ldots \ldots \ldots \ldots$ X

1. Teil. Gesamtverzeichnis der Abkürzungen in alphabetischer Reihenfolge ..... 1

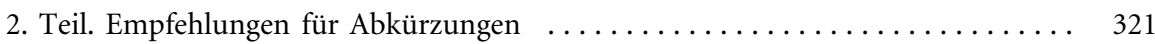

1. Empfehlungen für allgemeine Abkürzungen der Rechtssprache sowie für Behörden und Körperschaften $\ldots \ldots \ldots \ldots \ldots \ldots \ldots \ldots \ldots$

2. Empfehlungen für Abkürzungen von Gesetz- und Amtsblättern . . . . . 357

3. Empfehlungen für Abkürzungen von Zeitschriften und

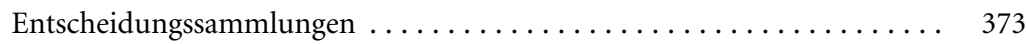

4. Empfehlungen für Abkürzungen von Gesetzen, sonstigen Rechtsvorschriften, Verwaltungsvorschriften u. ä. ............. 422

\section{Zur Beachtung für den eiligen Benutzer}

1. Rechts- und Verwaltungsvorschriften sind lediglich mit dem Datum der ersten Veröffentlichung aufgenommen worden. Spätere Änderungen wurden nicht berücksichtigt (siehe aber Nr. 2).

2. Neubekanntmachungen sind stets gebracht und durch »i. d. Bek. v.« gekennzeichnet.

3. Die Aufnahme einer Abkürzung für eine Rechts- und Verwaltungsvorschrift besagt nichts über deren Gültigkeit.

4. Für ältere, im allgemeinen nicht mehr benötigte Abkürzungen sind die früheren Auflagen weiterhin von Bedeutung.

5. Das Verzeichnis kann nicht allen möglichen Abkürzungsweisen Rechnung tragen. Daher sollte man auch vor und hinter der Buchstabenfolge suchen, deren Erklärung man wünscht.

6. Abkürzungen für Kommentare und Einzelwerke sind nicht aufgenommen.

7. Vom Gesetzgeber, von Verwaltungsinstitutionen bzw. bei Zeitschriften von Verlegern vorgesehene Abkürzungen sind durch Fettdruck hervorgehoben. 


\section{Regeln für die Bildung und den Gebrauch von Abkürzungen}

Wer je Abkürzungen (Kürzel) zu vergeben hat, sollte sich stets vor Augen halten, dass oberstes Ziel sein muss, das Gemeinte ohne Schwierigkeiten erschließen zu können. Aus diesem Grundsatz ergeben sich Art und Weise sowie Umfang der Abkürzung.

1. Abkürzungen von Rechts- und Verwaltungsvorschriften. - Ausgangspunkt für die Bildung einer Abkürzung ist grundsätzlich die Überschrift (z. B. Bundesnotarordnung). Ist für eine Rechts- und Verwaltungsvorschrift wegen ihrer längeren Benennung eine Kurzbezeichnung vorgesehen (z. B. »Bundesvertriebenengesetz« für »Gesetz über die Angelegenheiten der Vertriebenen und Flüchtlinge«) oder ist eine solche üblich (z. B. »Bundesbankgesetz« für »Gesetz über die Deutsche Bundesbank«), so ist diese Kurzbezeichnung für die Bildung der Abkürzung zu verwenden. Existiert eine Kurzbezeichnung nicht und ist eine solche auch schlechthin nicht zu bilden, so darf ausnahmsweise für die Abkürzung von einer sprachlich unmöglichen Form ausgegangen werden (z. B. Gesetz über die Angelegenheiten der freiwilligen Gerichtsbarkeit $=$ Freiwillige-Gerichtsbarkeit-Gesetz = FGG).

Die Normqualität gehört an den Schluss. Beim Beispiel FGG bezeichnet der letzte Buchstabe den Gesetzesrang der Regelung. Ebenso sind Rechtsverordnungen am Schluss durch ein $\mathrm{V}$ oder $\mathrm{VO}$ zu kennzeichnen.

2. Abkürzungen von Zeitschriftentiteln. - Bei der Bildung von Abkürzungen für Zeitschriften sowie Gesetz- und Amtsblätter und Entscheidungssammlungen ist von dem genauen Titel auszugehen. Der Titel darf für die Abkürzung nicht eigens verändert werden. Eine Abkürzung wie RFBl, die als Reichsfinanzblatt gedeutet werden muss, ist deshalb falsch gebildet; denn der Titel lautet »Amtsblatt der Reichsfinanzverwaltung«. Ein nur annähernd richtiger Titel ist aber ebenso unbrauchbar zum Suchen in Bücher- und Bibliothekskatalogen wie eine annähernd richtige Telefonnummer für den gewünschten Anschluss. Abkürzungen haben auch stets die gegebene Wortfolge zu berücksichtigen. (»Rechtsprechung der Hessischen Verwaltungsgerichte» sollte daher nicht HessVGRspr abgekürzt werden.) $\mathrm{Zu}$ missbilligen ist es auch, den Titel einer Zeitschrift zu ändern, die bisherige, für einen ganz anderen Titel gewählte Abkürzung aber weiterzuverwenden (z. B. StAZ für die Zeitschrift »Das Standesamt«, die vorher »Zeitschrift für Standesamtswesen» hieß).

3. Umfang der Abkürzung. - Mit wenigen Buchstaben sollten Rechts- und Verwaltungsvorschriften, Zeitschriften und Institutionen nur dann abgekürzt werden, wenn ihre Bekanntheit allgemein vorausgesetzt werden kann (BGB, NJW, BGH). Im Übrigen muss sich die Form der Abkürzung nach dem zu erwartenden Leserkreis richten. Sie kann kürzer gehalten sein, wenn nur ein kleiner Kreis von Sachkennern angesprochen werden soll.

4. Art der Bildung der Abkürzung. - Die sinntragenden Wörter bzw. Wortteile der Überschrift einer Rechts- und Verwaltungsvorschrift oder eines Titels sind durch Zusammenziehung (z. B. Ztg. für Zeitung) oder durch Weglassung der letzten Buchstaben (z. B. amtl. für amtlich) zu kürzen. Weniger wichtige Wörter, insbesondere Artikel und Verhältniswörter (Pronomina), können fortgelassen werden (z. B. Zeitschrift für Parlamentsfragen: ZParl). Artikel am Anfang eines Titels sollten sogar stets fortgelassen werden, weil ein 
abgekürztes D meist für Deutsch steht (z. B. DVBl bei Deutsches Verwaltungsblatt) und hierfür auch nicht entbehrt werden kann. DB als Abkürzung für »Der Betrieb« ist unglücklich gebildet. Adjektive sollten bei der Kürzung mit großem Anfangsbuchstaben geschrieben werden (z. B. ZVglRWiss = Zeitschrift für vergleichende Rechtswissenschaft), um den Anfang des neuen Wortes zu kennzeichnen, falls sich nicht eine abweichende Schreibweise für die Abkürzung durchgesetzt hat (z. B. AcP $=$ Archiv für die civilistische Praxis).

Buchstaben, die nicht am Anfang eines Wortes oder - bei zusammengesetzten Wörtern (Komposita) - des Wortteils stehen, sind bei der Kürzung klein zu schreiben (also nicht: STGB sondern StGB). Von dieser Regel sind nur eingebürgerte Abkürzungen ausgenommen (z. B. AnO = Anordnung, $\mathrm{VwV}=$ Verwaltungsvorschriften).

Von allgemein bekannten und üblichen Kürzungen sollte nicht abgewichen werden.

Abkürzungen sind grundsätzlich ohne Zwischenräume $\mathrm{zu}$ schreiben. Eine Trennung beim Zeilenende sollte vermieden werden, ist aber jeweils nach einem geschlossenen Bestandteil statthaft, wenn dadurch nicht einzelne Buchstaben abgesprengt werden ( $\mathrm{z}$. B. Rhein-Sch-UO = Rheinschiffs-Untersuchungsordnung).

5. Flexion der Abkürzungen. - Abkürzungen werden zumeist nicht flektiert (z. B.: des $\mathrm{BGB}$, nicht des BGBs). Soll zur Vermeidung in Missverständnissen gleichwohl die Flexionsendung wiedergegeben werden, so wird bei Abkürzungen, die mit dem letzten Buchstaben des Wortes enden, die Flexionsendung unmittelbar und vor dem Punkt (s.u.) angehängt (z. B.: Bde. = Bände). Der Plural ist sinngemäß zu bilden (RAe nicht RÄ = Rechtsanwälte). Die Pluralbildung durch Verdoppelung des letzten Buchstabens ist nur noch in Ausnahmefällen üblich (also: Art., nicht Artt. = die Artikel; aber immer noch häufig ff. $=$ folgende [Seiten, Paragraphen etc.]). Hier ist die Flexion zum Teil erforderlich (z. B.: die GmbHs, da sonst die Gefahr des Verwechselns mit dem Singular = die GmbH besteht).

6. Interpunktion bei Abkürzungen. - Eine besondere Uneinheitlichkeit und Unsicherheit besteht bei der Frage, ob hinter eine Abkürzung ein Punkt zu setzen ist oder nicht. Während die Juristen immer mehr dazu neigen, bei Abkürzungen jegliche Interpunktion zu unterlassen, gilt nach Duden nach wie vor die Grundregel, dass ein abschließender Punkt von Ausnahmen abgesehen dann zu verwenden ist, wenn die Abkürzung nicht gesprochen wird.

Nach diesen Regeln dürften wohl kurze Buchstabenfolgen wie ZPO, StGB und NJW ohne Punkt geschrieben werden können, nicht aber längere wie MSchG und EVRheinSchPatentV, die leichter durch den Volltext, für den sie stehen, als durch die gesprochenen Buchstaben wiedergegeben werden. Die Dudenregelung führt bei den Abkürzungen für Gerichte dazu, dass Buchstabenfolgen wie AG, LG, OLG, BGH ohne Punkt zu schreiben sind. Auch BVerfG wäre als sprechbar ebenso zu behandeln, während bei BVerwG die Schwierigkeit, ein am Ende einer Silbe stehendes w beim Sprechen deutlich herauszustellen, dazu führt, statt der Abkürzung lieber das Vollwort Bundesverwaltungsgericht zu benutzen. Neben der Grundregel der Unterscheidung nach der Sprechbarkeit der Abkürzung ist heute jedoch auch nach Duden anerkannt, dass sich im fachsprachlichen Bereich, insbesondere in den Rechtswissenschaften, immer mehr punktlose Abkürzungen durchsetzen.

Der Grund dafür liegt auch darin, dass es sich bei dem in vielen Fachsprachen verwendeten Kurzformen gar nicht um Abkürzungen im eigentlichen Sinne handelt. Nach den allgemeinen Interpunktionsregeln soll der Punkt hinter der Abkürzung das Fehlen von Buchstaben am Schluss oder im Inneren des Wortes kennzeichnen. Es ist deshalb s. $=$ siehe, Dr. $=$ Doktor zu schreiben. Um eine Wortabkürzung handelt es sich hingegen bei 
der Schreibung $\mathrm{m}=$ Meter und $\mathrm{Ra}=$ Radium gar nicht. Bei einer Abkürzung müsste man für Meter die Schreibweise M. erwarten. Ra für Radium wäre als Abkürzung einzusehen, wenn auch für Eisen Ei und für Kupfer $\mathrm{Ku}$ stünde. Stattdessen wird aber Eisen durch $\mathrm{Fe}$ (= Ferrum) und Kupfer durch $\mathrm{Cu}$ (= Cuprum) wiedergegeben. Das zeigt deutlich, dass wir es nicht mit Abkürzungen, sondern mit symbolhaften Schreibweisen zu tun haben, die man zum Unterschied gegen echte Abkürzungen als Sigel bezeichnen sollte.

Eine sigelmäßige Schreibung besteht im Allgemeinen auch bei den juristischen Abkürzungen. Das Wort "Strafgesetzbuch« müsste bei einer normalerweise durch Zusammenziehung (Kontraktion) gebildeten Abkürzung "Stgb." geschrieben werden. Üblich ist aber die Schreibung »StGB«. Eine echte Abkürzung läge auch vor, wenn »Ehegesetz« abgekürzt »Eheg.» geschrieben würde, jedoch wählt der Jurist »EheG«. Die sigelmäßige Kürzung wird oft auch aus der lückenlosen Schreibung mehrerer zusammengehöriger Wörter sichtbar (DVBl statt Dt.Verw.Bl.).

Dies letzte Beispiel zeigt, dass sich sigelmäßige und abkürzungsmäßige Schreibweise optisch leicht unterscheiden lässt und auch nebeneinander möglich ist. Die Wendung »am angegebenen Ort« lässt sich sowohl als Sigel - und dann ohne Punkt schreiben, nämlich »aaO«, oder als Abkürzung - und dann mit Punkt - »a.a.O.«. Das vorliegende Abkürzungsverzeichnis geht davon aus, dass in allen Fällen, so auch bei diesem Beispiel, der abkürzenden Schreibweise mit Punktfolge der Vorzug zu geben ist, insbesondere wenn es sich um Abkürzungen handelt, die nicht ausschließlich der Juristensprache angehören. Nur eine solche Entscheidung lässt sich konsequent durchhalten.

Eine generelle Punktlosigkeit ist nicht durchführbar, ohne Verwirrung zu stiften. Es lässt sich eben die Buchstabenfolge »so« nicht als »siehe oben« verstehen, wenn man die Punkte weglässt.

Die Interpunktionsregeln, wie sie diesem Verzeichnis zugrunde liegen, lassen sich wie folgt beschreiben:

a. Sigel werden ohne Schlusspunkt geschrieben. Sigel sind an ihrer besonderen, von der üblichen Schreibung der Wörter abweichenden Bildung erkennbar. Es kann sich dabei um Einzelwörter (z. B. StGB für Strafgesetzbuch) oder um Wortkomplexe handeln (z. B. GVBl für Gesetz- und Verordnungsblatt).

Ohne Punkt sind außerdem die als Sigel gedachten Buchstaben und Buchstabenfolgen der Allgemeinsprache zu schreiben (z. B. $\mathrm{m}=$ Meter, $\mathrm{S}=$ Schwefel, Ra $=$ Radium) sowie formelhafte Abkürzungen, die als Wörter verwendet werden (z. B. cif, fob).

b. Abkürzungen einzelner Wörter, insbesondere einzeln stehende Buchstaben als Abkürzungen, sind mit Schlusspunkt zu schreiben (z. B. s. = siehe, S. = Seite, Ges. = Gesetz). Ausnahmsweise kann V (= Verordnung) punktlos bleiben, wenn der Inhalt der Vorschrift anschließend wiedergegeben wird (z. B. V ü. d. Errichtung e. Beirates f. Ausbildungsförderung), der Buchstabe $\mathrm{V}$ im Text also nicht isoliert steht. 